\title{
EASy - Recuperação de Questões através de Metadados e Geração Automática de Instrumentos de Avaliação via $\mathbf{W e b}$
}

\author{
Renata Zanella ${ }^{1}$, Graciela Cristina Bernardes de Lima ${ }^{1}$, Bruna Antunes ${ }^{1}$, Paulo Blauth \\ Menezes ${ }^{1}$ \\ ${ }^{1}$ Instituto de Informática - Universidade Federal do Rio Grande do Sul (UFRGS) \\ Caixa Postal 15.064 - 91.501-970 - Porto Alegre - RS - Brazil \\ \{renataz ,gcblima, bafantunes, blauth\} @inf.ufrgs.br
}

\begin{abstract}
One of the most important characteristics of a Web learning environment on is the Evaluation task. Some of its functions are verify how much the student learned and supply feedback to the student and to the teacher. In this context, this paper presents EASy, a Web Evaluation System, designed to be integrated to XHA, a Web education environment based on Finite Automata with Output. EASy applies XHA's structural model, capitalizing the advantages presented in this model for Evaluations. The main focus of this system is in the questions's authoring , aiming automatic evaluations generation from configured metadata. This system also contemplates the functions of the evaluations, adaptive resources and feedback.
\end{abstract}

Keywords: EVALUATION, DISTANCE LEARNING, EDUCATIONAL SOFTWARES, AUTOMATA THEORY, WWW.

Resumo. Uma das características mais importantes em Sistemas de Ensino na Web é a tarefa Avaliação. Algumas de suas funções são verificar o quão e quanto o aluno aprendeu e fornecer feedback para o aluno e professor. Neste contexto, este artigo apresenta EASy, sistema de avaliações via Web, proposto para ser integrado ao XHA, ambiente de ensino via Web baseado em Autômatos Finitos com Saída. EASy aplica o mesmo modelo estrutural do XHA, capitalizando as vantagens apresentadas neste modelo para Avaliações. O foco principal deste sistema está na autoria de questões, visando geração automática de Avaliações a partir de metadados configuráveis. Este sistema contempla também as funções da avaliação, recursos adaptativos e feedback.

Palavras Chave: AVALIAÇÃO, ENSINO À DISTÂNCIA, SOFTWARES EDUCACIONAIS, TEORIA DOS AUTÔMATOS.

\section{Introdução}

A Web tornou-se um meio importante para a disseminação do conhecimento, pois oferece uma diversidade de informações, dados e pesquisas disponibilizadas através de ferramentas ou ambientes de ensino-aprendizagem, que podem ser acessados a qualquer hora e de qualquer lugar.

No processo de ensino-aprendizagem, encontra-se a avaliação, considerada uma tarefa importante, pois possibilita aos professores receberem informações sobre $\mathrm{o}$ aprendizado do aluno e verificar se os objetivos do curso foram atingidos. O Tema Avaliação é bastante amplo, o que possibilita a exploração e aplicação de diversos instrumentos, técnicas e funções de avaliação, resultando em sistemas que podem seguir diferentes caminhos, alguns com ênfase maior no processo de autoria de avaliações [Cardoso, 2001], [Fischer e Steinmetz, 2000] outros mais focados no acompanhamento e direcionamento dos alunos através de feedback, relatórios e monitoração [Otsuka, 2002]. 
Este artigo apresenta o EASy, "Evaluation Automatic Generation System for Web based on Hyper-Auotmaton". O objetivo principal deste trabalho é fornecer uma ferramenta de apoio ao professor, reduzindo assim, o esforço despendido no processo de criação e correção das avaliações, visando uma dedicação maior no processo de avaliação dos resultados apresentados pelos alunos nas avaliações.

O restante deste artigo está organizado da seguinte maneira: a seção 2. apresenta as principais características pedagógicas e tecnológicas de Avaliações via Web; a seção 3. apresenta o ambiente de ensino XHA, Sistema a ser integrado com este trabalho; a seção 4. apresenta o EASy, trabalho proposto; a seção 5 apresenta os agradecimentos; e a última seção apresenta as conclusões e trabalhos futuros.

\section{Avaliação via Web}

A avaliação é uma das mais importantes tarefas em todo processo de ensino e aprendizagem, além de causar maior impacto na maneira e conseqüência do aprendizado dos estudantes [Kwok, 1999].

A automatização de parte do processo de confecção e submissão de testes é uma alternativa eficiente e de custo relativamente baixo para alcançar um rápido feedback. Se utilizada com moderação e combinada com outras formas de testes, a avaliação online pode fornecer ainda outras maneiras de avaliar o desempenho do estudante com uma significativa redução de sobrecarga para professores [Tinoco, 97].

A avaliação compreende por três funções: diagnóstica, formativa e somativa [Bloom, 1983]. Cada avaliação pode apresentar uma função específica, complementar das restantes. A avaliação formativa é a que ocorre ao longo do processo de aprendizagem. Um de seus objetivos é correção de falhas do processo educacional através de feedback. A avaliação somativa tem caráter classificatório e ocorre ao final de um processo. Por fim a avaliação diagnóstica, que tem como objetivo verificar se os pré-requisitos necessários para o curso são atendidos pelo aluno.

Para cada uma das funções, encontram-se instrumentos de avaliação. Segundo [Haydt, 2000], instrumentos de avaliação são os recursos que podem ser utilizados para a obtenção de informações relevantes ao processo de avaliação. Entre os predominantes estão os Testes Objetivos e as Provas Dissertativas.

Este trabalho optou por implementar Testes Objetivos, que segundo Medeiros é um instrumento de medida composto de questões tão precisamente especificadas, que cada qual só admita uma resposta, previamente definida, o que lhe assegura a impessoalidade do julgamento e inteiro acordo entre examinadores diferentes, cuja opinião não influi nos resultados. É importante salientar que os testes objetivos serão aplicados para as três funções da avaliação, cabendo ao professor escolher a função e o instrumento adequado.

Neste trabalho, estão disponíveis três instrumentos de avaliações: Provas Objetivas, Listas de Exercícios e Questionários. Todos compostos por questões objetivas, projetadas para otimizar dados/informações relevantes ao processo de avaliação. Busca-se capitalizar as vantagens apresentadas por testes objetivos como correção automática, avaliação de vários objetivos ao mesmo tempo, eliminação do aspecto subjetivo da correção e trabalhar em cima das desvantagens como elaboração difícil e demorada, e facilitador da "cola" [Haydt 00]. 


\section{XHA - eXtensible Hyper-Automaton}

XHA é um ambiente de ensino via Web, baseado em Autômatos Finitos com Saída , que apresenta diversos recursos como material instrucional independente da estrutura de navegação, facilidade de reuso de páginas em diversos cursos, com eliminação da redundância, capitalizados do Sistema Hyper-Automaton [Machado et al., 2000]. Além das vantagens relacionadas com modelo aplicado, existe a tecnologia empregada: XML, XSL, JSP e Java, que apresentam funcionalidades próprias para aplicações Web, possibilitando um Sistema mais flexível na disponibilização do material, suporte a recursos de links não convencionais, redução do número de caracteres incompatíveis entre navegadores e o aumento de recursos didáticos dos cursos criados no XHA.

O modelo Hyper-Automaton está baseado em Autômatos Finitos com Saída (máquina de Moore $^{I}$ e Mealy $^{2}$ ), onde cada página de avaliação/conteúdo do curso corresponde a um estado (Moore) ou a uma transição (Mealy). A vantagem é que a estrutura dos autômatos com saída permitem a criação do material hipermídia de forma independente do autômato em si, reuso de conteúdo de forma completa ou parcial em diversos cursos (autômatos), eliminado a redundância de informações e criações de páginas.

Visando as vantagens apresentadas pelo modelo, a tecnologia empregada no XHA, a importância da tarefa avaliação e a obtenção de um Ambiente de EnsinoAprendizagem, foi proposto o EASy, Evaluation Automatic Generation System for Web based on Hyper-Automaton.

\section{EASy}

EASy tem como objetivos principais: (1) suprir algumas funcionalidades desejadas em sistemas de avaliação via Web como flexibilidade na autoria de avaliações, reuso de questões, recursos de edição para questões e avaliações, avaliações adaptativas e personalizadas e (2) fornecer um sistema de avaliação para o ambiente de ensino XHA.

Buscou-se neste trabalho capitalizar os recursos tecnológicos e o modelo estrutural empregados no XHA, onde o autômato ${ }^{3}$ que define uma avaliação é uma estrutura formal preestabelecida e neste, como anteriormente definido em [Machado et. al, 2000], determina toda a sua estrutura navegacional.

Tendo-se como foco principal deste trabalho à autoria, buscou-se reunir um conjunto de características que estão sendo utilizados em sistemas similares [Cardoso, 2001], [Brusilovsky e Miller, 1999], [Choren, 1998] como banco de questões, informações sobre o desempenho do aluno para o professor e para o aluno, e contribuir com recursos que permitem flexibilizar o processo de autoria através (1) do modelo Hyper-Automaton empregado, (2) do conjunto de metadados utilizados para a geração de avaliações automáticas e personalizadas e (3) de recursos adaptativos.

Desta forma, são apresentadas as subseções: a Arquitetura; a Utilização do Sistema e os Ambientes de utilização do Sistema para alunos e professores.

\footnotetext{
${ }^{1}$ A Máquina de Moore possui uma segunda função, que gera uma palavra de saída (que pode ser vazia) para cada estado da máquina.

${ }^{2}$ A Máquina de Mealy é um Autômato Finito modificado de forma a gerar uma palavra de saída para cada transição 


\section{Arquitetura}

A arquitetura proposta para este trabalho baseia-se no conceito Three-Tiered Architecture (arquitetura em três camadas). As camadas ajudam a identificar, definir e, potencialmente, restringir como os elementos de cada camada interagem uns com os outros [Ambler, 1998], [Rumbaugh et al., 1994].

A arquitetura em três camadas de serviços é constituída pela camada de apresentação, pela camada de negócio e pela camada de dados. A Figura 1 apresenta a arquitetura para o sistema proposto.

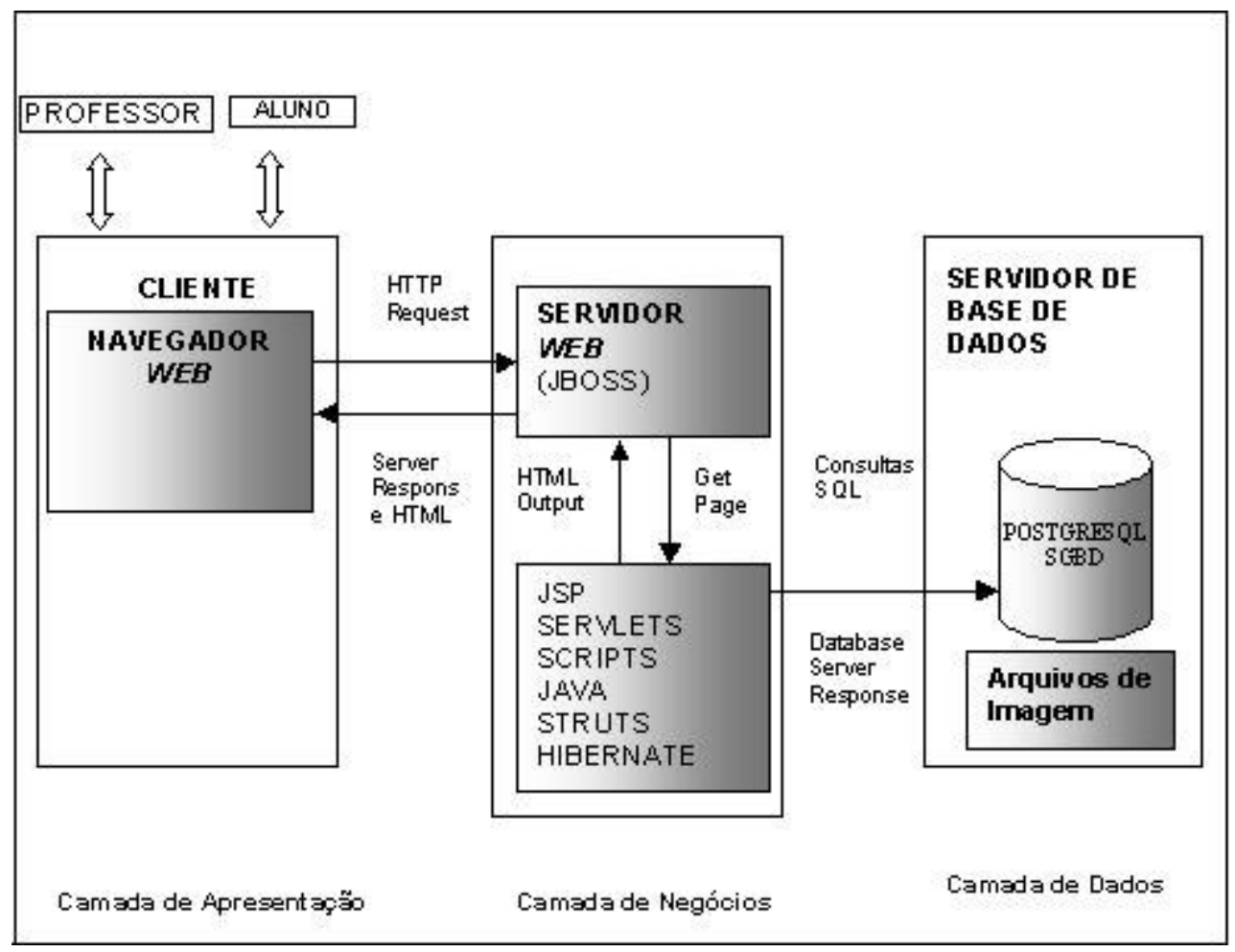

Figura 1. Arquitetura do Sistema

A camada de apresentação corresponde pela interface entre o sistema e o usuário. Nesta camada estão as telas de manipulação do sistema e à entrada de dados fornecidos pelos usuários. A camada de negócios é responsável por oferecer todas as funcionalidades do sistema. Ela é composta pelo Jboss (Servidor Web+ EJB) e todos os programas Java responsáveis por executar as requisições realizadas pelo usuário ao Sistema e pela comunicação com a base de dados, retornando então a saída em páginas HTML geradas dinamicamente. A camada de dados do sistema é composta pelo banco de dados Postgresql e um diretório de arquivos. No banco de dados estão armazenados todos os dados relativos ao sistema de avaliações, como por exemplo, questões, gabaritos, Avaliações como Hyper-Automaton, avaliações resolvidas, metadados, etc

\section{Utilização do Sistema}


EASy apresenta dois ambientes de utilização, um para professores e outro para alunos. Após a autenticação no sistema é disponibilizado o ambiente apropriado, ou seja, o ambiente gerenciador de avaliações, para professores, e o ambiente realizador de avaliações, para alunos. A Figura 2 apresenta o modelo funcional do EASy.

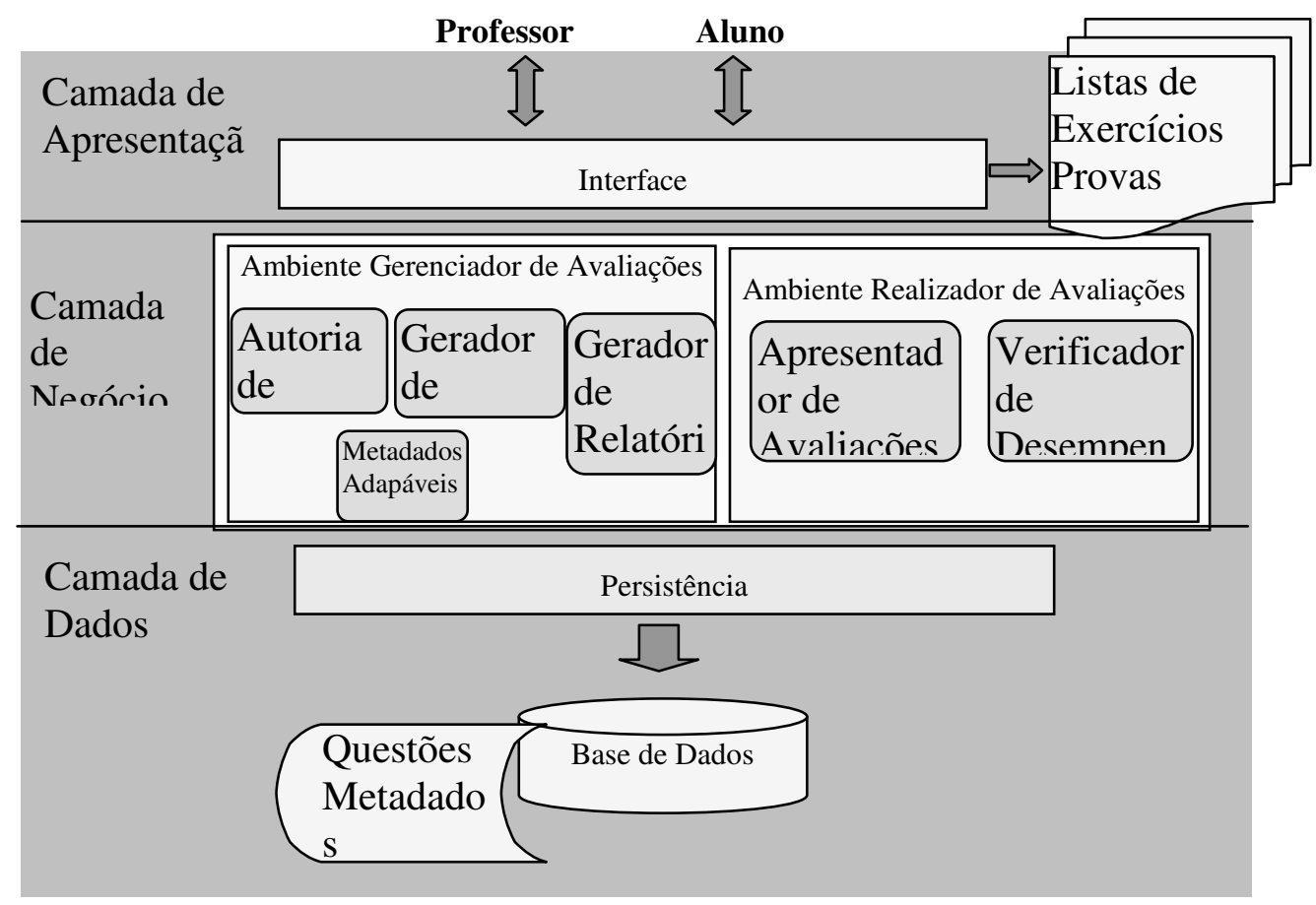

Figura 2. Modelo Funcional do EASy

\section{Ambiente Gerenciador de Avaliações}

Este ambiente é destinado aos professores e compreende pelos seguintes módulos: autoria de questões, gerador de avaliações, avaliações adaptativas, metadados adaptáveis e gerador de relatórios. Neste artigo serão apresentados os módulos principais do sistema: Módulo de Autoria de Questões, Módulo de Geração de Avaliações e Módulo Gerador de Relatórios.

Módulo de Autoria de Questões - neste módulo, o professor pode criar, editar e excluir as questões através de uma interface transparente ao usuário. Ao criar uma questão, o professor pode incluir o feedback de erro para cada questão ou para cada alternativa. A figura 3 apresenta uma tela de criação de questões. 


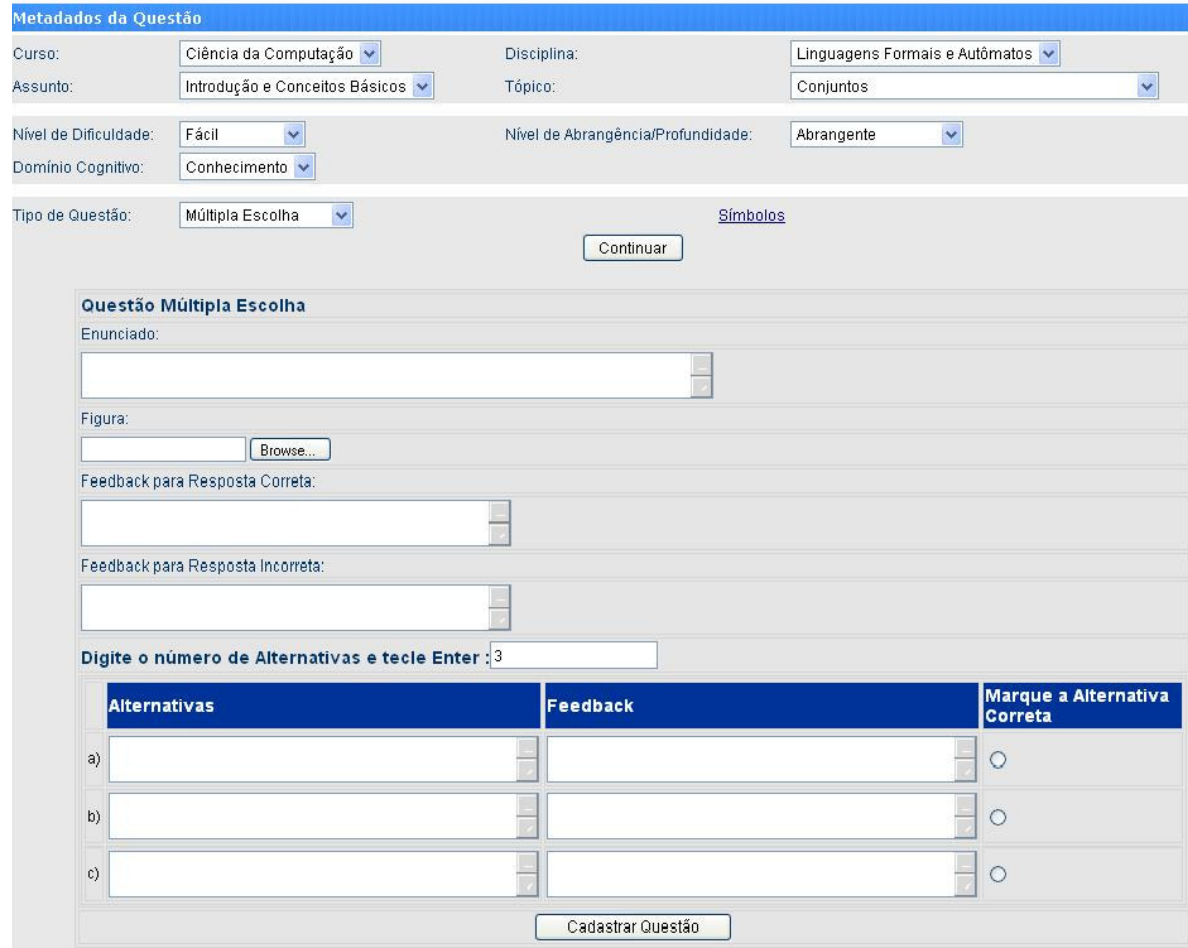

Figura 3. Tela de criação de Questões

Módulo de Geração de Avaliações - a geração de avaliações pode ser automática ou semi-automática. Na geração automática o usuário solicita os tópicos relacionados com o objetivo da avaliação como mostra a Figura 4 e em seguida o sistema apresente um conjunto de metadados caso o professor queira um grau maior de filtro das questões como mostra a Figura 5.

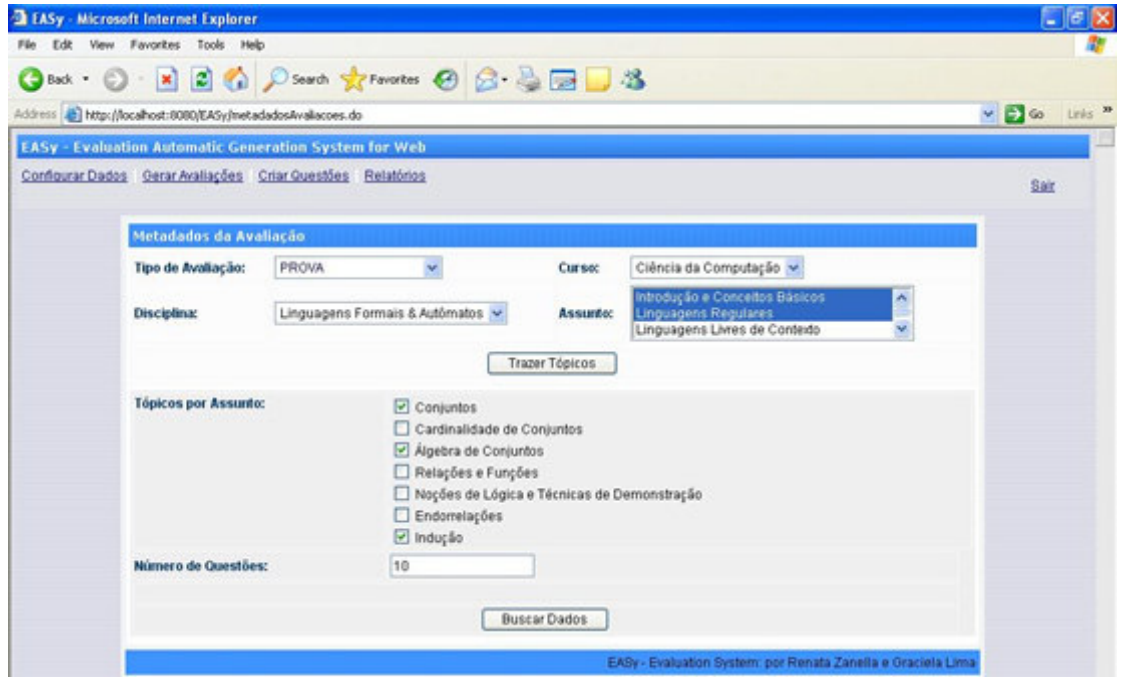

Figura 4. Tela de Geração de Avaliações 


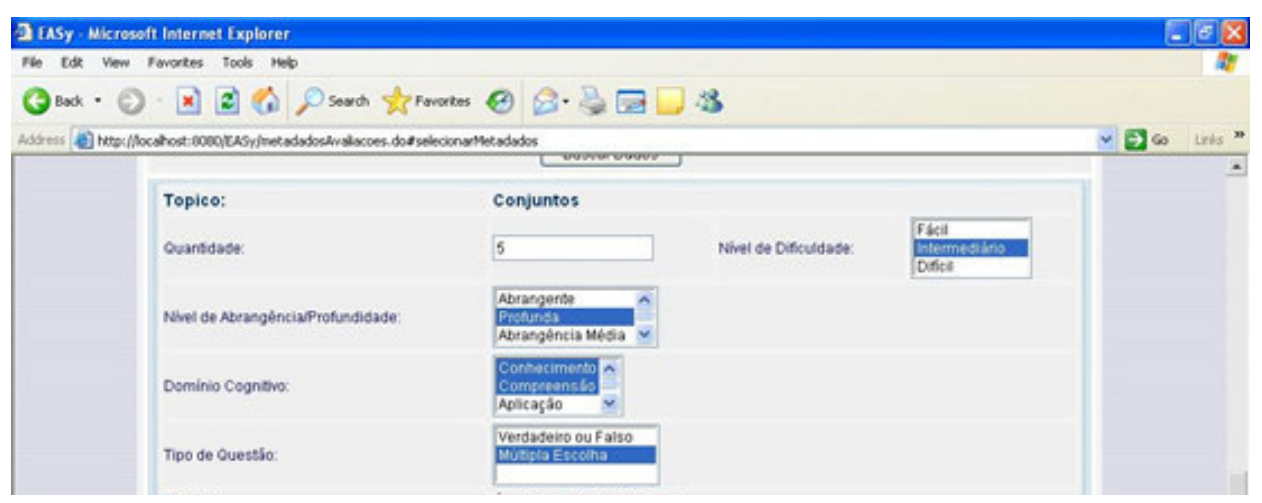

Figura 5. Metadados das Questões

Nas avaliações semi-automáticas, o professor pode interagir com a avaliação, editando a sequiência das questões como mostra a Figura 6. Este módulo é responsável também pela edição e exclusão das avaliações.

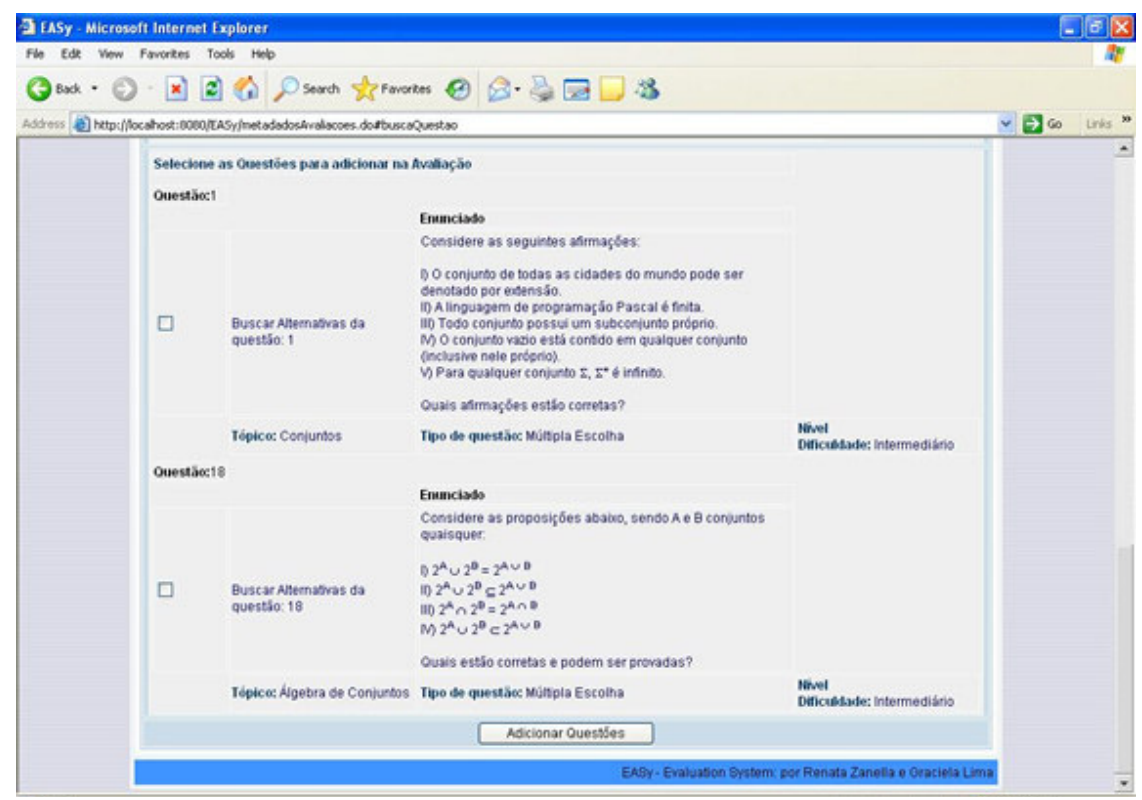

Figura 6. Tela de Seleção das questões para inserir na Avaliação

Módulo Gerador de Relatórios - este módulo é responsável pela geração de relatórios sobre o desempenho dos alunos. A partir destes relatórios, o professor pode acompanhar o rendimento do aluno ou da turma e verificar se seus objetivos estão sendo atingidos. A Figura 8 apresenta um relatório das questões erradas dos alunos.

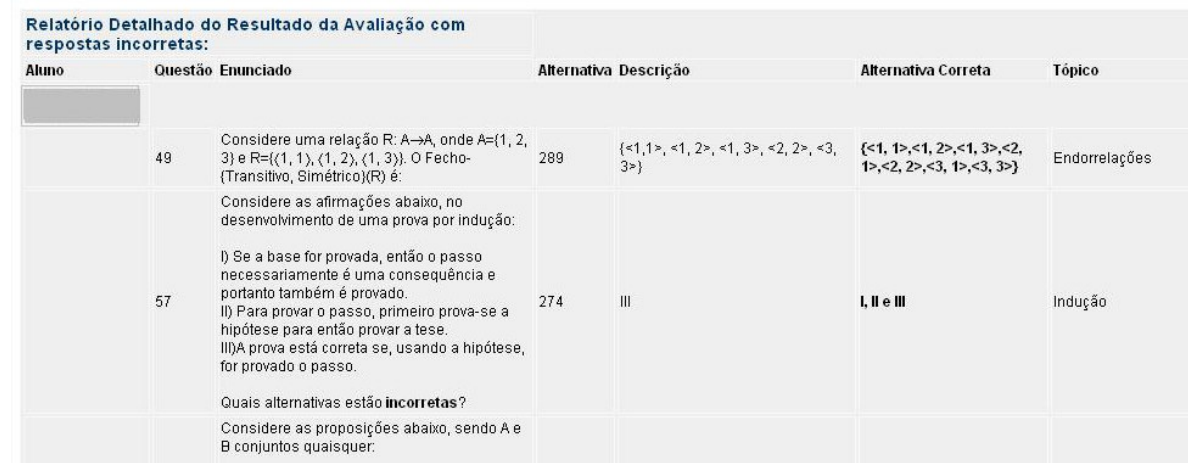

Figura 8. Relatório sobre os erros dos alunos e o tópico relacionado 


\section{Ambiente de Realização de Avaliações}

Este ambiente é responsável por apresentar as avaliações para serem realizadas e o desempenho destes a partir da correção das avaliações pelo sistema. Este ambiente compreende pelo módulo de apresentação de avaliações e módulo verificador de desempenho.

Módulo de Apresentação das Avaliações - o módulo de apresentação é responsável por entregar ao aluno os instrumentos de avaliação: lista de exercícios, provas ou questionários, as quais poderão ser realizadas a partir da autenticação do aluno no sistema e da disponibilização destas para o mesmo. Após realizar a avaliação, o aluno a envia e esta é corrigida pelo sistema, em seguida o aluno recebe o feedback sobre a avaliação. Quando for uma lista de exercícios, o aluno recebe o feedback de erro, ao pressionar o botão corrigir questão, este compreende por explicar porque a resposta dele está errada e fornecer algumas dicas para reforçar o estudo. Caso o aluno esteja fazendo uma prova, o feedback de erro será fornecido no final da prova com informações sobre as questões certas e erradas, orientação de estudo a partir dos erros e a nota do aluno. Todos estes dados são armazenados no banco de dados e podem ser recuperados depois pelo professor através do gerador de relatórios e pelo aluno através do módulo verificador de desempenho.

Módulo Verificador de Desempenho - este módulo é responsável por apresentar informações sobre as avaliações realizadas aos alunos. Eles podem obter relatórios sobre seu desempenho nas avaliações e as sugestões para reforçar o estudo. A figura 9 apresenta um exemplo de feedback dos resultados do aluno na avaliação. São apresentadas questões que ele respondeu erradamente com a alternativa correta e o tópico relacionado.

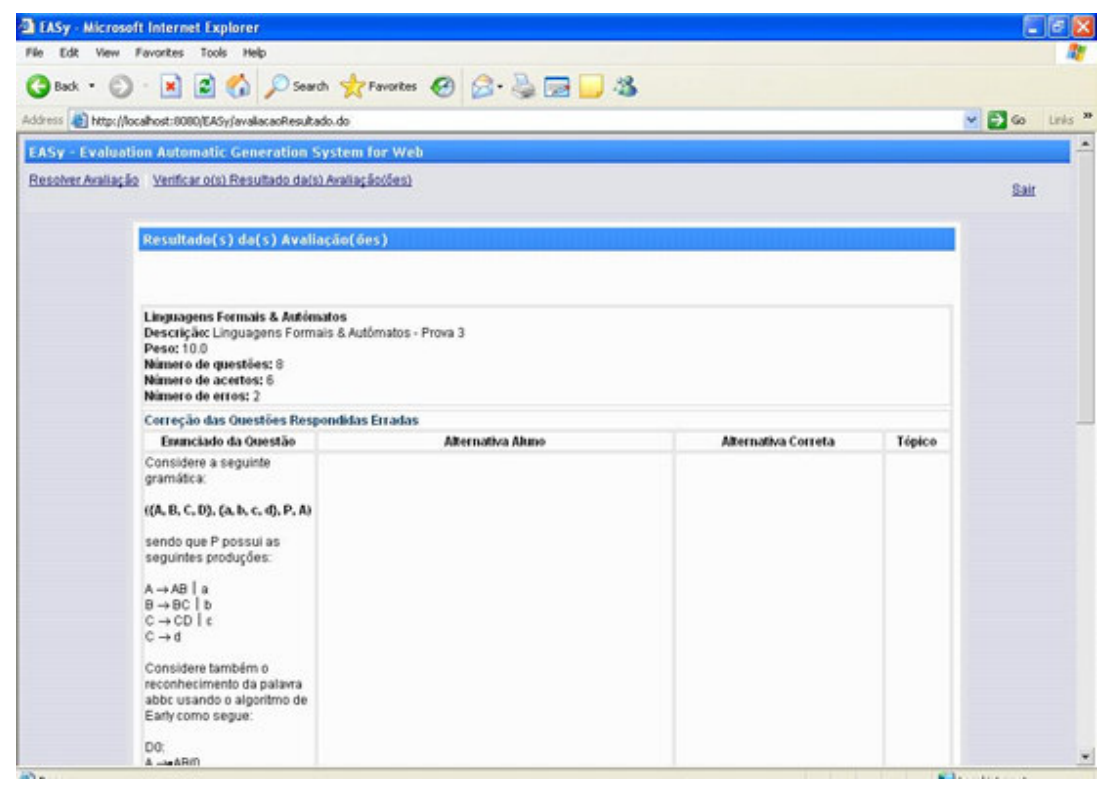

Figura 9. Tela de Feedback para o Aluno

\section{Integração do EASy com o XHA}

A integração dos módulos foi realizada na camada de persistência e na base de dados, que foi estendida para armazenar os dados referentes às avaliações sem interferir no modelo Hiper-Autômato. 


\section{Considerações Finais e Trabalhos Futuros}

Este trabalho contribui para o processo de ensino-aprendizagem via $W e b$, pois apresenta um sistema que auxilia o professor no processo de autoria, apresentação e manutenção de avaliações através de uma interface gráfica transparente ao usuário, reuso de questões e material instrucional independente da estrutura de navegação. Além destas características, o sistema fornece feedback para o professor e para o aluno, promovendo a orientação do aluno com informações complementares sobre o assunto da avaliação e informações sobre o desempenho do aluno para o professor; apresenta aspectos teóricos da avaliação, permitindo que o professor escolha aquele que achar mais adequado; oferece recursos adaptativos, importantes para adequar o estudo ao ritmo de aprendizado do aluno e possibilita a integração com o ambiente de ensino XHA.

Como trabalhos futuros estão previstas, a automatização dos metadados adaptáveis e aplicação de técnicas adaptativas no processo de ensino-aprendizagem, onde se pretende adaptar o conteúdo conforme o conhecimento ou não conhecimento do aluno a partir da realização das avaliações. A adaptação está prevista com a integração deste sistema com o XHA.

\section{Agradecimentos}

Este trabalho está sendo parcialmente financiado pelo CNPq (Projetos HoVer-CAM, GRAPHIT, E-Automaton) e FINEP/CNPq (Projeto Hyper-Seed).

\section{Referências}

Ambler, S. W. (1998). Análise e Projeto Orientados a Objeto, volume II: Seu Guia para Desenvolver Sistemas Robustos com Tecnologia de Objetos. Ed. Infobook, 1998.

Bloom, B. S. et al. (1983). Manual de avaliação formativa e somativa do aprendizado escolar ". 1 ed. São Paulo: Pioneira, 1983. 307 p".

Brusilovsky. P., Miller, P. (1999). Web-based Testing for Distance Education. In: Webnet World Conference on WWW and Internet.

Cardoso, R., LIMA, J. V. (2001). AvalWeb - Sistema interativo para gerência de questões e aplicação de avaliações na Web. In: Anais do VII Workshop de Informática a Escola, Fortaleza, Brasil, julho de 2001.

Choren, R. et. al. (1998). QUEST - An Assessment Tool for Web-Based Learning. In: Webnet 1998. Proceedings ... Orlando, 1998.

Fischer, S., Steinmetz, R. (2000). Automatic creation of exercises in adaptive hypermedia learning systems. ACM Hypertext, 2000 : 49-55.

Haydt, R. C. (2000). Avaliação do processo ensino-aprendizagem. 6 ed. São Paulo: Ática.

Kwok, R.C.; MA, J. (1999). Use of a group support system for collaborative assessment. Computers \& Education, Great Britain, v. 32, p. 109-125, Feb. 1999.

Machado, C. C.; Federizzi, G. L.; Menezes, P.B. (2001). Definition and Application of Rules for the Adequate Designing of XML Documents for the Hyper-Automaton System. In: Internacional Workshop On Database And Expert Systems Applications - DEXA, 12., 2001, Munich - RDA. Proceedings... [S.1.: s.n], 2001.

Machado, J. P. Morais, C.T.Q. de, Menezes, P. B., Reis, R.A.L.(2000). Structuring Web Course Pages as Automata: Revising Concepts. Recherce D'informations Assistee Par Ordinateur, Riao, 2000, Paris. Proceedings... Paris: Centre de Hautes Etudes Internationales 
d'Informatique Documentaires, Center for the Advanced Study of Information Systems, 2000, v.1, p.150-159.

MEDEIROS, E. B. Provas Objetivas, discursivas, orais e práticas: Técnicas de Construção. Rio de Janeiro: Ed. Fundação Getúlio Vargas, 1986.

Morais, C. T. Q., Menezes, P. F. Blauth, Reis, R. Modeling for Automatic Generation of Exercises and Evaluations. In: ICECE'2000: International Conference on Engineering and Computer Education, 2000, São Paulo. Cooperative Network for Engineering and Computer Education Development : proceedings. São Paulo: SENAC/SP, 2000. v.1. p.100 - 105.

Otsuka, J. L.; Lachi, R. L.; Ferreira, T. B.; Rocha, H. V. (2002). Suporte à Avaliação Formativa no Ambiente de Educação a Distância TelEduc. In: Anais do VI Congresso Iberoamericano de Informática Educativa (IE2002), Vigo, Espanha 20-22 novembro.

Rumbaugh, J.; Blaha, M.; Premerlani, W.; Frederick, E.; Lorensen, W. (1997). Modelagem e Projetos Baseados em Objetos. Ed. Campus, 1997.

Tinoco, L. C. et. al. (1997). On-line evaluation in WWW-based Courseware. In: Communications of ACM. p. 194-198.SIGCSE 97. 1997. 